\title{
EFFETS DE LA VAGOTOMIE INTRATHORACIQUE PARTIELLE SUR LA SURVIE ET LA CROISSANCE DU MOUTON
}

\author{
M. DUSSARDIER \\ Avec la collaboration technique de J. L. Durel et de Colette Lavenet. \\ Station de Recherches de Physiologie animale, \\ Centre national de Recherches zootechniqes, Jouy-en-Josas.
}

\section{SOMMAIRE.}

Chez le Mouton, la section intrathoracique du nerf pneumogastrique gauche (en arrière du récurrent) et celle du tronc vagal inférieur sont parfaitement tolérées. Elles n'altèrent même pas la croissance. Par contre la section intrathoracique du tronc vagal supérieur entraine des troubles graves et souvent mortels.

Au cours de diverses expériences, nous avons sectionné dans le thorax certaines branches du pneumogastique chez des moutons et des boucs. L'étude de la mortalité post-opératoire nous a conduit, en ce qui concerne l'inocuité des différentes sections, à des conclusions qui s'accordaient mal avec les données bibliographiques. Nous avons donc entrepris une étude spéciale de ce problème en utilisant des lots d'animaux homogènes, ce qu'aucun auteur n'avait fait jusqu'à présent.

\section{TECHNIQUE}

Vingt agneaux de même origine, pesant de $3^{2}$ à $40 \mathrm{~kg}$, ont permis de constituer quatre lots à peu près identiques au départ.

Les animaux des lots I, II et III ont subi la section intrathoracique respectivement du pneumogastrique gauche (en arrière de l'émergence du récurrent), du tronc vagal inférieur (en arrière de l'anastomose des deux nerfs inféricurs) du tronc vagal supérieur (en arrière de l'anastomose des deux nerfs supérieurs). Chez les animaux du lot IV, les nerfs ont été découverts, mais non sectionnés. Les opérations se sont échelonnées sur neuf jours.

\section{RÉSULTATS}

\section{I. - COMPARAison DES lots I, II ET IV.}

Tous les animaux ont survécu à 1'opération. Ils ont été abattus 75 jours après.

Le tableau résume un certain nombre de données numériques. Nous ne trouvons nulle part une différence statistiquement significative entre les différents lots. La 
vagotomie unilatérale et la section du tronc vagal inférieur ont donc été parfaitement tolérées.

TABI,EAU

Effet des opérations chirurgicales sur la croissance des animaux et sur le poids de leurs estomacs.

\begin{tabular}{|c|c|c|c|c|}
\hline Poids moyen en $\mathrm{kg}$ & $\begin{array}{c}\text { Lot I vagroto- } \\
\text { mie gauche }\end{array}$ & $\begin{array}{l}\text { Lot II vagoto- } \\
\text { mie inférieure }\end{array}$ & $\begin{array}{l}\text { Lot IV } \\
\text { Témoins }\end{array}$ & $\begin{array}{c}\text { Interprétation de la différence } \\
\text { des moyennes (test } \mathrm{F} \text { ) }\end{array}$ \\
\hline Poids vif avant l'opération & 36,2 & 36,2 & 36,2 & Différence des moyennes nulle \\
\hline Poids vif à l'abattage .... & 47,8 & 46,1 & 46,5 & Non significatif $F<1$ \\
\hline Poids de la carcasse..... & 24,4 & 22,8 & $2: 3,7$ & Non significatif $\mathrm{F}<1$ \\
\hline Poids du rumen vide .... & 0,812 & 0,714 & 0,752 & Non significatif $P>0,05$ \\
\hline Poids du réseau vide..... & $0,11_{18}$ & 0,137 & 0,135 & Non significatif $\mathrm{F}<1$ \\
\hline Poids du feuillet vide .... & 0,100 & 0,087 & 0,086 & Non significatif $\mathrm{P}>0,05$ \\
\hline Poids de la caillette vide. & 0,191 & 0,211 & 0,185 & Non significatif $F<1$ \\
\hline
\end{tabular}

\section{II. - DEVENIR DES ANIMAUX DU LO'T III.}

'Trois des animaux ont présenté des vomissements dans les jours qui ont suivi 1'opération. Ils sont morts respectivement 4, 6 et I2 jours après l'opération, d'une broncho-pneumonie par corps étrangers ayant pour origine l'aspiration intra-trachéale des matières alimentaires vomies.

Les deux autres animaux ont survécu. Ils ont été fortement météorisés dans les

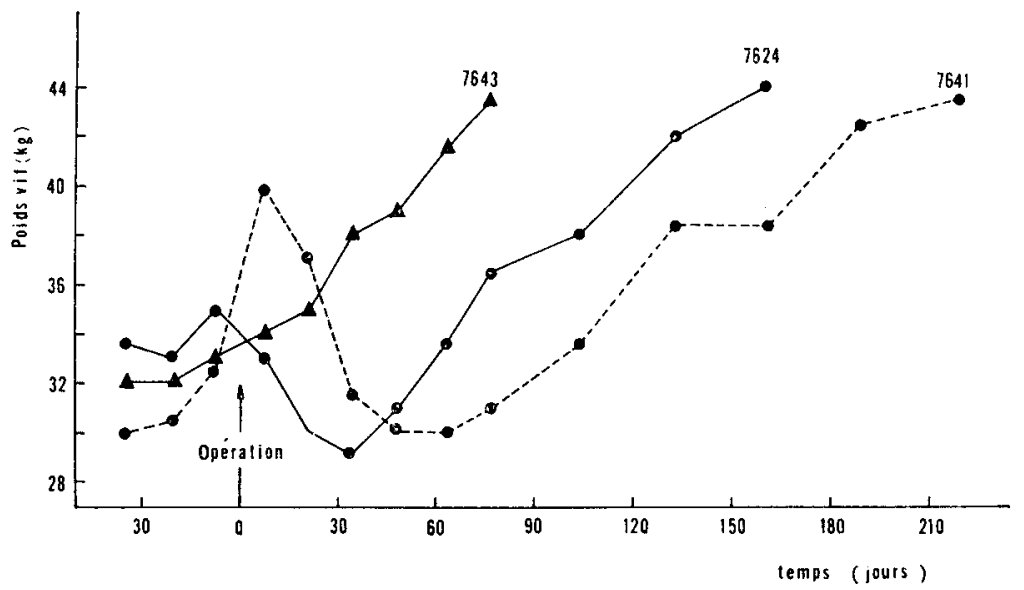

FIG. - Courbe de poids comparative de deux moutons (7624 el 7641) qui ont subi une section du tronc vagal supérieur, et d'un animal du lot témoin (7643).

jours et les semaines qui ont suivi l'opération. Par la suite, la météorisation est devenue moins grave mais n'a jamais disparue : la récupération totale n'est pas possible.

Le graphique montre 1'évolution du poids de ces deux animaux et d'un animal témoin du lot IV. La section du tronc vagal supérieur a provoqué un amaigrissement et un retard de croissance important. L'augmentation du poids de 1'animal 764I, qui est passé en $I_{5}$ jours de 32,5 à $40 \mathrm{~kg}$ traduit une stase gastrique considérable.

Les deux survivants ont été munis neuf mois après l'opération d'une fistule de la 
région antérieure du rumen selon la technique de DUSSARDIER (I96I). Les contractions du réseau paraissaient normales. Nous ne pouvons pas dire si les variations de pression dont le rumen était le siège correspondaient à des contractions normales, car il est difficile de réaliser, pour cet organe, des conditions d'enregistrement constantes. L,es animaux étaient capables de ruminer.

A l'examen histologique l'extrémitié périphérique du tronc vagal supérieur de l'un des deux animaux, mort au bout de I3 mois, est apparue totalement dégénérée

\section{TISCUSSION}

Nos expériences montrent que la section du pneumogastrique gauche ou celle du tronc vagal inférieur, sont parfaitement tolérées, tandis que celle du tronc vagal supérietır a des conséquences graves. Ces résultats concordent parfaitement avec ce que nous pouvions prévoir d'après les données anatomiques (voir HABEL, I956). Selon celles-ci, en effet, il semble qu'aucun organe ne doive être totalement dénervé par la vagotomie unilatérale ou la section du trone vagal inférieur. Au contraire, la section du tronc dorsal doit dénerver la plus grosse partie du rumen ; or la motricité intrinsèque de cet organe est trop faible pour remuer son volumineux contenu.

Cependant, sauf en ce qui concerne la tolérance parfaite de la vagotomie unilatérale, nos résultats ne sont pas en accord avec ceux des auteurs qui ont déjà étudié le problème.

Pour MAxgol, et pour WEIss, la section du tronc vagal supérieur est parfaitement tolérée, tandis que celle du tronc inférieur produit des troubles graves. HoFLund a sectionné le tronc vagal inférieur chez sept animaux; cinq n'ont extériorisé aucun troubleou seulement des troubles mineurs; deux sont morts vingt jours après l'opération. Pour D. Duncan la section du tronc vagal inférieur et celle du tronc supérieur sont bien tolérées.

Ces divers auteurs ont réalisé des sections intra-abdominales, ce qui risque d'entraîner les causes d'erreur suivantes : création d'adhérences péritonéales (signalées par HoFluND) qui peuvent par elles-mêmes perturber le fonctionnement digestif ; oubli de certaines branches nerveuses, ou au contraire section de branches qui auraient du être respectées ; enfin confusion des troncs supérieur et inférieur qui semble avoir été faite par Mangol, comme l'indiquent Phim,ipson, Duncan et Habei.

Nous avons évité ces causes d'erreur en réalisant des sections intrathoraciques et nous pouvons donc être très affirmatif en ce qui concerne l'inocuité de la section du tronc vagal inférieur. Cependant, la section intra-thoracique du tronc vagal supérieur dénerve la partie terminale de l'œsophage. Nous ne pouvons donc pas affirmer que tous les troubles que nous avons observés sont dus à l'énervation des estomaes. Il resterait à faire une étude comparative des effets de la section sus- ou sous-diaphragmatique du tronc vagal supérieur.

$$
\text { Rę̧u en mai } 1960 .
$$

\section{SUMMARY.}

THE EFFECT OF PARTIAL INTRATHORACIC VAGOTOMY ON THE SURVIVAL AND GROWTH OF SHEEP

In the sheep, the intrathoracic removal of the left vagus nerve (behind the recurrent) and of the lower vagal trunk are tolerated perfectly. The growth is not even altered. On the other hand, the intrathoracic removal of the upper vagal trunk results in serious and often fatal disorders. 


\section{RÉFÉRENCES BIBLIOGRAPHIQUES}

Duncan D., 1953. The effects of vagotomy and splanchnotomy on gastric motility in the sheep. J. Physiol. London, 119, I $57-169$.

Dussardier M., I $96 r$. Technique de fistulation de la région antérieure du rumen par la voie transthoracique chez les petits Ruminants. Ann. Biol, anim. Bioch. Biophys., 1, I13-1 I6.

HABEL R. E., I956. A study of the innervation of the ruminant stomach. Cornell Vel., 46, 555-628.

Hoflund S., I940. Untersuchungen über Störungen in den Funktionen der Wiederkäuermagen durch Schădigungen des N. Vagus Verursacht. Svensk Veterinäritskrift, suppl. au tome 54 (Isaac Marcus, Stockholm 332 pages).

MANGOLD E., I929. Handbuch der Ernährung und des Stoffwechsels der landwirtschaftlichen Nutztiere. tome 2. Julius Springer, Berlin, p. 193-199.

Phillipson A. T., I942. Digestion in the ruminant. Ph. D. Thesis, Cambridge. (cité par DunCaN).

WeIsS K. E., I953. Physiological studies on eructation in ruminant. Onderstepoort J. Vet. Kes., 26, 25 I283 . 